\title{
RECONFIGURABLE ION SELECTIVE SENSOR ARRAY ENABLED BY DIGITAL MICROFLUIDICS
}

\author{
A. Farzbod ${ }^{l}$ and H. Moon ${ }^{l}$ \\ ${ }^{1}$ The University of Texas at Arlington, USA
}

\begin{abstract}
This paper presents a proof of concept demonstration of a reconfigurable ion selective electrodes (ISE) array enabled by an electrowetting on dielectric (EWOD) digital microfluidics, for the first time. The on-chip preparation of an ISE array includes electroplating $\mathrm{Ag}$ followed by forming $\mathrm{AgCl}$ layer by chemical oxidation and forming a thin layer of arbitrary ion-selective membrane liquid on a sensor electrode. The calibration curve of fabricated ISE shows complete agreement with conventional ones. The proposed device with the capability of on-chip ISE fabrication has many advantages such as automation simplicity, minimal membrane consumption, longer lifetime and easy integration in lab-on-a-chip (LOC) platforms.
\end{abstract}

\section{INTRODUCTION}

In an EWOD digital microfluidics, liquids are manipulated in droplet form by applying electrical potential to series of electrodes with dielectric layer on top [1]. Many superior capabilities of digital microfluidics over channel-based microfluidics, such as multiplexing capability, have been demonstrated. The precise control over working liquids and reagents makes them suitable for lab on a chip and micro analysis platforms [2]. In general, lab-onchip (LOC) devices consist with two main units - the liquid handling unit and the chemical analysis unit. Over the past decades, there has been significant improvement in both areas. However, complete integration of different fluid handling and sensor technologies remains as complex tasks. There has been efforts to integrate optical sensors in digital microfluidics [3], but such integration is not IC compatible nor easy to use. In general, having two (or more) units with different working principles in one microsystem makes the system bulky and less attractive for commercial use.

With improvement in solid-state micro-electrochemical sensors and growth of bio electrochemical sensors in the past two decades [4][5][6], electrochemical analysis began to draw attentions again due to their simplicity and easy fabrication. Various methods support electrochemical sensing - cyclic voltammetry, amperometry, potentiometry and impedance spectroscopy, etc. Recently, an amperometric sensor was integrated into EWOD device to analyze ferrocene methanol and dopamine [8]. Also Liu et al reported integration of conductometric sensors on microfluidic LOC devices [9]. However, integrating potentiometric sensors, wherein the signal is not dependent on the area of the sensor, has not been popularly considered due to oxidation of electrode which results in shorter lifetime of the sensor [10].

To address the challenge of short sensor lifetime, in this paper, we report an ion selective potentiometric sensor fabricated on-chip with the help of EWOD. This new method allows users of LOC device to reconfigure the sensor array online without any interruption or the disassembling of the device while increasing the lifetime of the sensor. In addition, it will enable on-demand sensor functionality of LOC devices - type of sensors are not predesignated but user can determine it at the time of use.

This present work focuses on testing the proposed on-chip sensor fabrication and evaluating the performance of the fabricated sensor by comparing it with that of conventional ISEs.

\section{EXPERIMENTAL \\ Material}

$\mathrm{Au} / \mathrm{Cr}$ coated wafer was used to fabricated EWOD electrodes and the seed layer of ISEs. S1813 (MICROPOSIT) was used as the photoresist for patterning and mask layer in different stages of fabrication. SU-8 2005(Micro-Chem) was used as dielectric layer. The solution of Teflon AF1600S (DuPont) powder dissolved in Fluorinert FC-40 (Sigma-Aldrich) was spin coated to form hydrophobic layer. 1025 RTU (TECHNIC INC) was used for Ag electroplating solution. $\mathrm{HCl}$ analytical reagent grade was used for formation of $\mathrm{AgCl}$ layer. For Potassium ion selective membrane which contains $1 \mathrm{wt} \%$ of potassium ionophore I, $0.5 \mathrm{wt} \%$ potassium tetrakis(4-chloropheny) borate, 49.5 wt \% bis(2ethylhexyl)sebacate and 49 wt \% PVC were used; these materials were purchased from Sigma-Aldrich. Tetrahydrofuran (THF-T3974) was used as an organic solvent and carrier liquid for potassium ion selective membrane. For acquiring the calibration curve, different molarities of $\mathrm{KCl}$ solution $(1 \mu \mathrm{M}-1 \mathrm{M})$ were prepared by dissolving potassium chloride (Sigma-Aldrich) powder in Deionized water.

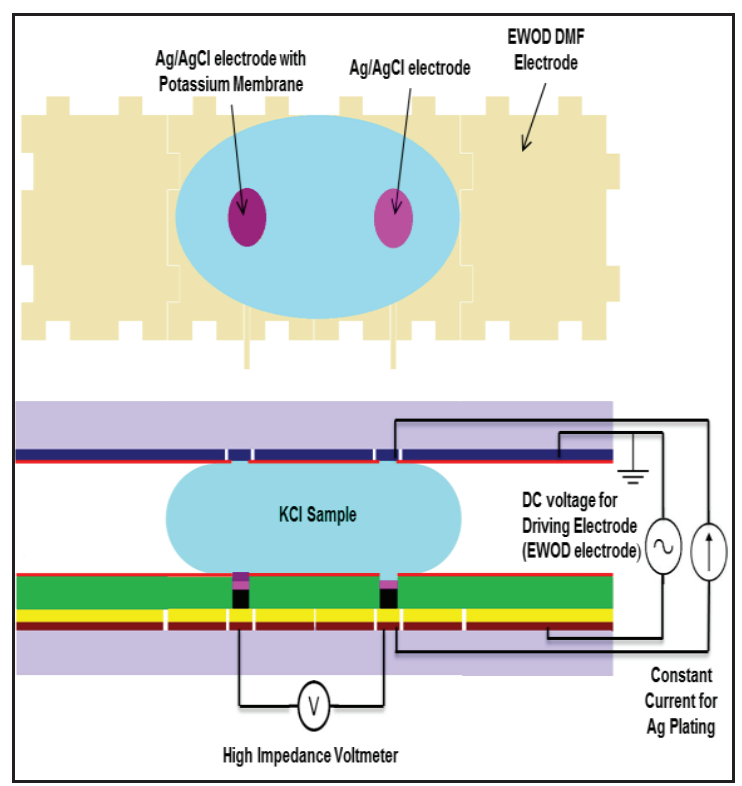

Figure 1: two-chip configuration of EWOD DMF integrated with ISE.

\section{Design \& configuration of EWOD-ECC}

Figure 1 shows the configuration of the EWOD electrochemical cell (EWOD-ECC). The top plate is coated with indium tin oxide (ITO) to provide a ground electrode for EWOD motion as well as a cathode electrode for electroplating. The bottom plate contains 
patterned gold $\mathrm{(Au}$ ) electrodes for EWOD operating electrodes as well as sensor electrodes. Although the use of Au electrodes is common to cyclic voltammetric and amperometric sensors, $\mathrm{Au}$ electrodes cannot deliver a stable voltage in potentiometric applications. Whereas $\mathrm{Ag} / \mathrm{AgCl}$ electrodes can establish the required stability for the voltage measurement, thus $\mathrm{Ag} / \mathrm{AgCl}$ electrodes are commonly used as reference electrodes in electrochemical sensors. The significant improvement in the fabrication of $\mathrm{Ag} / \mathrm{AgCl}$ electrodes through electroplating and chemical anodization methods lends even higher stability for EMF measurement [11] yet the short lifetime of $\mathrm{Ag} / \mathrm{AgCl}$ electrodes still exist. Therefore, in this study, we present the DMF platform where we fabricate our $\mathrm{Ag} / \mathrm{AgCl}$ electrode on-demand and on-chip as demonstrating the renewal of worn-out reference electrodes surfaces. For liquid handling, we used six reservoirs with the capability of dispensing droplets $(\sim 650 \mathrm{~nL})$ and 45 electrodes $(2 \times 2$ $\mathrm{mm}^{2}$ each) for EWOD driving electrodes. For sensing, we designed four electrochemical cells with sensor electrodes $(400 \mu \mathrm{m}$ diameter) integrated with EWOD electrode. The hydrophilic-tohydrophobic ratio (i.e. sensor to EWOD electrode area) was 3.1\%; this ratio is critical in order not to hinder the droplet motion.

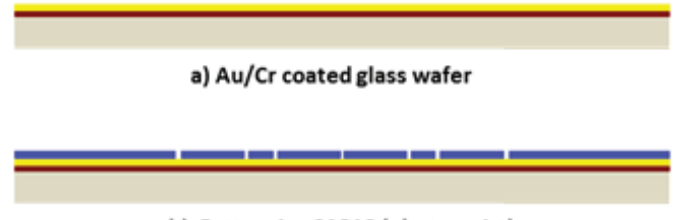

b) Patterning $\$ 1813$ (photoresist)

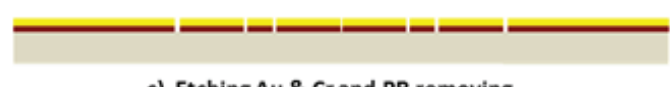

c) Etching Au \& $\mathrm{Cr}$ and PR removing

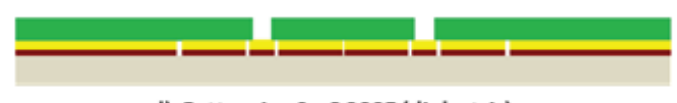

d) Patterning Su-82005 (dielectric)

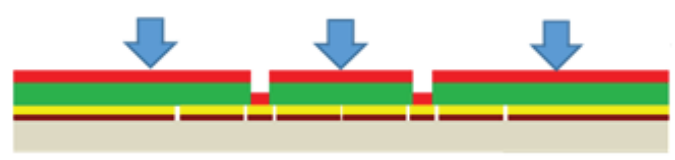

e) Teflon coating and wetting of Teflon surface with RIE

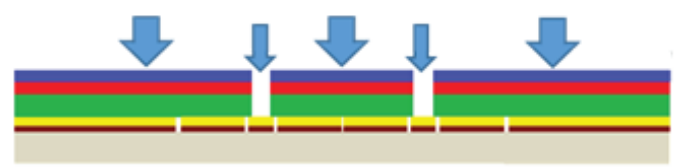

f) PR patterning and RIE for selective etching
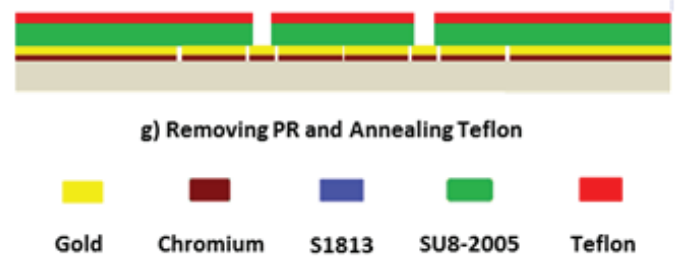

Figure 2: Fabrication process of sensor electrodes integrated in the bottom plate of EWOD digital microfluidic chip.

\section{EWOD-ECC fabrication}

Figure 2 shows the fabrication of EWOD-ECC. The electrodes of EWOD device and sensor electrode were patterned by standard lithography followed by wet chemical etching of $\mathrm{Au} / \mathrm{Cr}$ layer. Su8-2005 was then spin coated and flood-exposed to form a dielectric layer over the EWOD electrodes with the thickness of $5 \mu \mathrm{m}$. Afterwards, a Teflon layer $(300 \mu \mathrm{m})$ was spincoated and baked to form a hydrophobic coating. This was followed by reactive ion etching treatment of Teflon surface for 5 seconds at 140 Watts and $30 \mathrm{sccm}$ Ar rate. This step makes the Teflon surface ready for the spin-coat of S1813 to form RIE etching mask. Then, after selectively etching the Teflon layer over the sensor electrodes for 45 minutes with the power of 200 Watts and $30 \mathrm{sccm}$ Ar rate, the remaining PR layer was exposed to UV light (flood exposure) to get weakened and removed by dissolving in acetone solution. Finally, after annealing the chip in $200{ }^{\circ} \mathrm{C}$ oven for one hour, the Teflon surface recovered its hydrophobicity properties and got ready for operation.

\section{On-chip ISE fabrication and calibration}

To carry out the on-chip fabrication of $\mathrm{Ag} / \mathrm{AgCl}$ sensor electrodes (Figure 1), a droplet $(\sim 650 \mathrm{~nL})$ of $\mathrm{Ag}$ plating solution was dispensed from the reservoir and brought over the sensing site by EWOD microfluidic actuation (Step 1 in Figure 3). Electroplating was driven at $12 \mathrm{~mA} / \mathrm{cm}^{2}$ for 300 seconds. To avoid $\mathrm{Ag}+$ depletion, plating solution droplets were replenished. Similarly, a droplet of $0.1 \mathrm{M} \mathrm{HCl}$ solution was dispensed and brought over the Ag-plated electrodes (Step 2 in Figure 3) and applied to the surface for $45 \mathrm{~s}$ to form $\mathrm{AgCl}$ layer. Lastly, a droplet was dispensed from membrane liquid reservoir and brought over a sensor electrode to form the layer of an ion selective membrane (ISM) (Figure 4(c)) and Step 3 in Figure 3). Due to the wettability contrast, the liquid membrane was pinched-off at the hydrophilic opening on the sensor electrode (Figure 5). After evaporation of THF, a thin layer of the gel-like membrane has been formed over sensor electrode.

Electromotive force (EMF) was measured with high input impedance $\left(10^{12} \Omega\right)$ using HP $34401 \mathrm{~A}$ multimeter at room temperature $\left(21{ }^{\circ} \mathrm{C}\right)$ in an electrochemical cell fabricated on the chip.

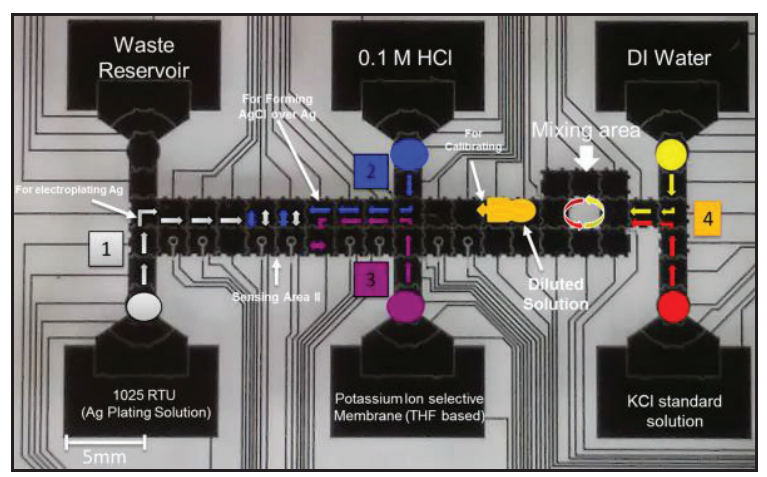

Figure 3: Fabrication on-chip and serial dilution and calibrating procedure. Step (1): Ag electroplating, Step (2): chemical oxidation of Ag layer, Step (3): forming a thin layer of ISM on a sensor electrode and step, and Step (4): serial dilution for calibrating sensor. 

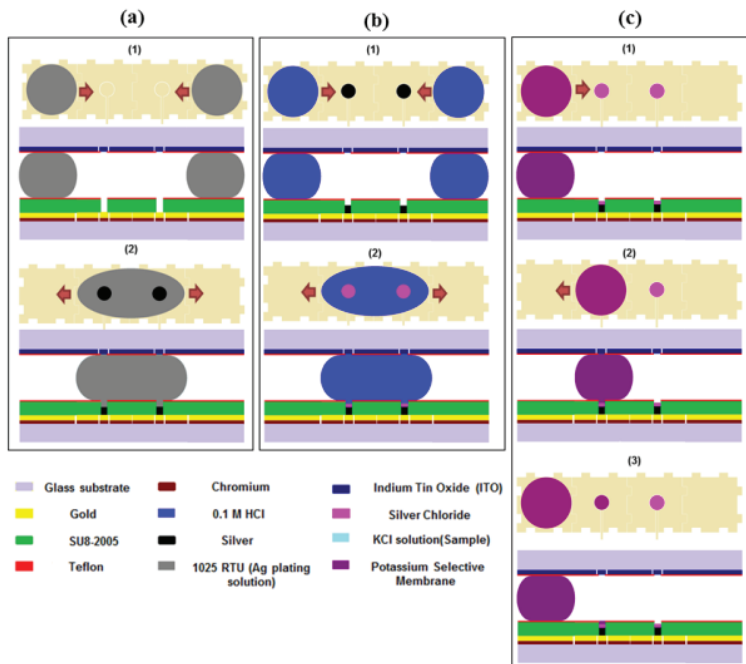

Figure 4: Process flow of on-chip fabrication of ISE. (a) Silver electroplating, (b) chemical oxidation of Ag layer with $\mathrm{HCl}$ to form $\mathrm{AgCl}$ layer, and (c) forming of a thin layer of potassium selective membrane on sensor electrode.
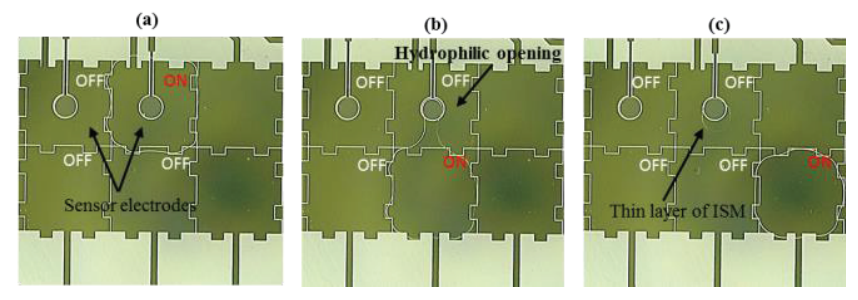

Figure 5: Ion-selective membrane (ISM) pinching-off process; (a) an ISM liquid droplet brought over a sensor electrode by EWOD motion, (b) the ISM droplet being driven away from the sensor electrode while a part of ISM wetting the hydrophilic opening over the sensor electrode (c) a thin layer of ISM on sensor electrode after the completion of pinch-off.

\section{RESULTS \& DISCUSSION}

The theory of potentiometric sensors is based on NicolskiiEisenman formalism (1) which derived from Nernst equation:

$$
E_{\text {cell }}=E_{\text {cell }}^{0} \square(R T / z F) \ln \left(a_{i}(I)\right)
$$

Where, $a_{i}(I)$ is the primary ion activity in the sample.

We used $\mathrm{Ag} / \mathrm{AgCl}$ for both electrodes and used potassium ISM on one of the electrodes, therefore our measured electromotive force is related to the activity of potassium in the solution.

The standard calibration curve for the potassium ion selective sensor was performed on-chip. Serial dilution of standard $\mathrm{KCl}$ solution by DI water was done on the designated mixing area (Step 4 in Figure 3) to prepare 11 different molarity $\left(10^{-6}-10^{-1} \mathrm{M}\right)$ solutions. Droplets of each molarity were brought over the on-chip fabricated ISE; then electromotive force was measured (Figure 6). Figure 7 shows the Nernstian response to solutions as indicating that the on-chip fabricated ISE work with complete agreement with typical ISEs.

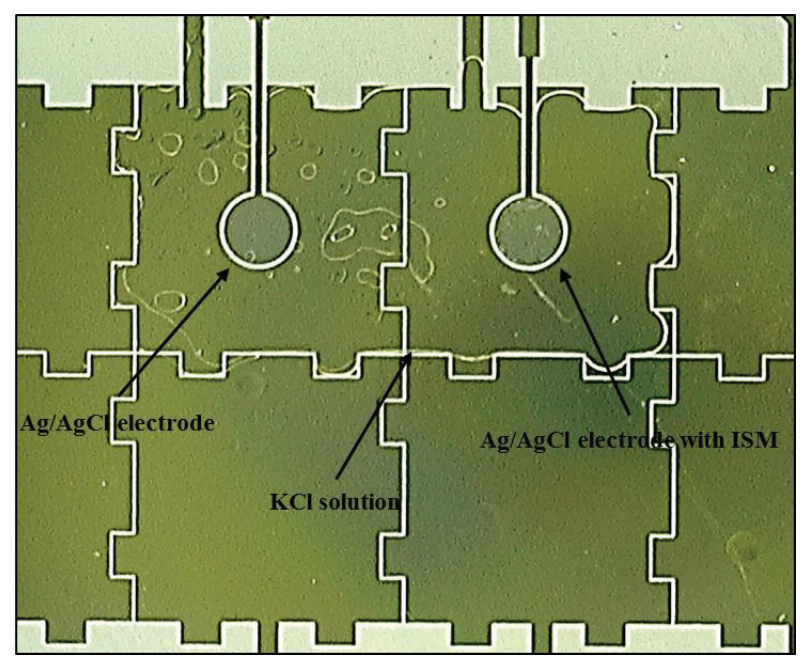

Figure 6: Measuring electromotive force on EWOD

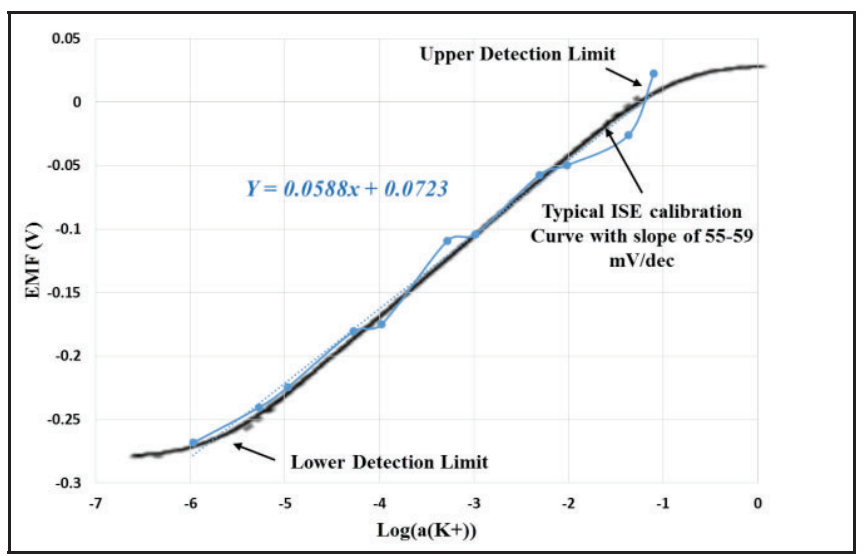

Figure 7: Nernstian response to different molarity of $\mathrm{KCl}$ solutions

\section{CONCLUSION}

This successful demonstration promises that the proposed reconfigurable ISE can provide a sensor device where a user can define and form desirable ISEs instantaneously at the time of use. Simultaneous (and serial) detection of multiple analytes will be available. Also, ISEs will be renewable by adding dissolving membrane and washing electrode steps followed by another membrane formation step.

The proposed platform solved the main challenge of the potentiometric sensor by adding lifetime to sensor electrodes while adding a reliable and reproducible analysis unit to lab-on-a-chip devices. This method of fabrication can pave the path to low cost and compatible home-use sensors with using benefits of EWOD such as ease of use and automation and minimal consumption of reagents.

\section{REFERENCES}

[1] S. K. Cho, H. Moon, and C. J. Kim, "Creating, transporting, cutting, and merging liquid droplets by electrowetting-based actuation for digital microfluidic 
circuits," J. Microelectromechanical Syst., vol. 12, no. 1, pp. 70-80, 2003.

[2] M. J. Jebrail and A. R. Wheeler, "Let's get digital: Digitizing chemical biology with microfluidics," Curr. Opin. Chem. Biol., vol. 14, no. 5, pp. 574581, 2010.

[3] K. Zhang, G. Tao, X. Zeng, W. Sheng, and J. Zhou, "Compact and portable chemiluminescence detector for glucose," Proc. Int. Conf. ASIC, 2013.

[4] H. Arida, Q. Mohsen, and M. Schöning, "Microfabrication, characterization and analytical application of a new thin-film silver microsensor," Electrochim. Acta, vol. 54, no. 13, pp. 3543-3547, May 2009.

[5] D. Grieshaber, R. MacKenzie, J. Vörös, and E. Reimhult, "Electrochemical Biosensors - Sensor Principles and Architectures," Sensors, vol. 8, no. 3, pp. 1400-1458, 2008.

[6] M. Odijk, E. J. van der Wouden, W. Olthuis, M. D. Ferrari, E. a. Tolner, a. M. J. M. van den Maagdenberg, and a. van den Berg, "Microfabricated solid-state ion-selective electrode probe for measuring potassium in the living rodent brain: Compatibility with DC-EEG recordings to study spreading depression," Sensors Actuators, B Chem., vol. 207, pp. 945-953, 2014.

[7] B. Kintses, L. D. van Vliet, S. R. A. Devenish, and F. Hollfelder, "Microfluidic droplets: New integrated workflows for biological experiments," Curr. Opin. Chem. Biol., vol. 14, no. 5, pp. 548555, 2010.

[8] Y. Yu, J. Chen, and J. Zhou, "Parallel-plate lab-ona-chip based on digital microfluidics for on-chip electrochemical analysis," J. Micromechanics Microengineering, vol. 24, no. 1, p. 015020, Jan. 2014.

[9] G. Liu, K. Smith, and T. Kaya, "Implementation of a microfluidic conductivity sensor - A potential sweat electrolyte sensing system for dehydration detection," 2014 36th Annu. Int. Conf. IEEE Eng. Med. Biol. Soc. EMBC 2014, pp. 1678-1681, 2014.

[10] M. Novell, M. Parrilla, G. a. Crespo, F. X. Rius, and F. J. Andrade, "Paper-based ion-selective potentiometric sensors," Anal. Chem., vol. 84, pp. 4695-4702, 2012.

[11] B. J. Polk, A. Stelzenmuller, G. Mijares, W. MacCrehan, and M. Gaitan, "Ag/AgCl microelectrodes with improved stability for microfluidics," Sensors Actuators B Chem., vol. 114, no. 1, pp. 239-247, Mar. 2006.

\section{CONTACT}

Ali Farzbod, tel: +1-682-521-2553; Ali.farzbod@mavs.uta.edu 